\title{
Carbon nanoparticle-guided intraoperative lymph node biopsy predicts the status of lymph nodes posterior to right recurrent laryngeal nerve in cNO papillary thyroid carcinoma
}

\author{
Yuwei Ling^, Lina Zhang, Kaifu Li, Ye Zhao, Jing Zhao, Luyao Jia, Yajun Wang, Hua Kang \\ Center for Thyroid and Breast Surgery, Department of General Surgery, Xuanwu Hospital, Capital Medical University, Beijing, China \\ Contributions: (I) Conception and design: H Kang; (II) Administrative support: H Kang; (III) Provision of study materials or patients: K Li, J Zhao, \\ H Kang; (IV) Collection and assembly of data: Y Ling, L Zhang, Y Zhao; (V) Data analysis and interpretation: Y Ling; (VI) Manuscript writing: All \\ authors; (VII) Final approval of manuscript: All authors. \\ Correspondence to: Hua Kang. Center for Thyroid and Breast Surgery, Department of General Surgery, Xuanwu Hospital, Capital Medical University, \\ Beijing 100053, China. Email: kanghua@xwh.ccmu.edu.cn.
}

\begin{abstract}
Background The lymph nodes in the right central compartment can be divided into 2 parts by the right recurrent laryngeal nerve (RLN), and there is a lack of an accurate and convenient method for assessing metastases in the lymph node posterior to the right recurrent laryngeal nerve (LN-prRLN) in cN0 thyroid papillary carcinoma patients.
\end{abstract}

Methods: Patients diagnosed with cN0 thyroid papillary carcinoma and underwent intraoperative carbon nanoparticle-guided lymph node biopsy from January 2017 to November 2020 at the Center for Thyroid and Breast Surgery of Xuanwu Hospital were retrospectively analyzed. The intraoperative frozen section examination and postoperative LN-prRLN status should have been comprehensively recorded. The participants were divided into the LN-prRLN positive group and LN-prRLN negative group according to their recorded LN-prRLN status.

Results: In total, 189 cases (LN-prRLN positive group, $\mathrm{n}=30 ; \mathrm{LN}$-prRLN negative group, $\mathrm{n}=159$ ) were included in the analysis. The univariate and multivariate regression analyses revealed that the number of metastatic lymph nodes during intraoperative lymph node biopsy was the potential predictor for LNprRLN metastasis [odds ratio (OR): 1.320, 95\% confidence interval (CI): 1.057 to $1.649, \mathrm{P}=0.014$ ]. The receiver operating characteristic (ROC) curve showed that the area under the curve (AUC) reached 0.7 upon a combined analysis of multiple lymph node statuses located at pre-laryngeal (Delphian), pre-tracheal, and para-tracheal lymph nodes ipsilateral to the tumor in predicting the metastasis of LN-prRLN, and the cutoff value was 0.5 .

Conclusions: Number of metastatic lymph nodes in intraoperative biopsy was an indicator of LN-prRLN metastasis in cN0 thyroid carcinoma patients. Patients staging in cN0 with negative intraoperative lymph node status might be considered not to require LN-prRLN dissection during central lymph nodes dissection.

Keywords: Papillary thyroid carcinoma (PTC); carbon nanoparticles; intraoperative lymph node biopsy; lymph node posterior to the right recurrent laryngeal nerve; risk factors

Submitted Dec 27, 2020. Accepted for publication Mar 21, 2021.

doi: $10.21037 /$ gs-20-920

View this article at: http://dx.doi.org/10.21037/gs-20-920

\footnotetext{
^ ORCID: 0000-0001-8487-3738.
} 


\section{Introduction}

Papillary thyroid carcinoma (PTC) is one of the most common subtypes of differentiated thyroid carcinoma (DTC) and accounts for approximately $85 \%$ of thyroid carcinomas (1). Although PTC is identified as a kind of indolent tumor, positive lymph node metastasis is present in $20-90 \%$ of PTC patients (2), leading to local recurrence and distant metastasis (3). However, prophylactic central neck dissection has remained controversial due to the increased risk of hypoparathyroidism and recurrent laryngeal nerve injury (4-6). The 2015 American Thyroid Association (ATA) management guidelines for DTC did not recommend routine prophylactic central lymph node dissection (CLND) and illustrated that thyroidectomy without prophylactic CLND might be more appropriate for T1 or T2, noninvasive, and clinically node-negative PTC (cN0) (7). The Chinese guidelines for DTC recommended prophylactic CLND should be performed with proper protection of the parathyroid and recurrent laryngeal nerve (RLN), and prophylactic CLND was also accepted for $\mathrm{cN} 0$ PTC in the Japanese guidelines (8). Our clinical practice routinely performed prophylactic CLND to obtain more accurate tumor pathological staging.

From an anatomical point of view, the right central compartment's lymph nodes could be divided into 2 parts by the right RLN due to the ascension of the right RLN through the central compartment, which differs from the anatomical structure on the left side. As a result, there are several lymph nodes posterior to the RLN, which tend to be omitted during CLND (9). The lymph nodes on the posterior side of the right RLN are usually identified as LN-prRLN. The American Head and Neck Society Consensus Statement published in 2017 states that the right LN-prRLN should be routinely dissected during right CLND (9). Undoubtedly, dissection of LN-prRLN can significantly increase the potential risk of RLN injury, lymphatic leakage, pleural injury and increase the operating time. Also, ultrasonography (US) has fairly poor sensitivity in assessing lymph node metastases in the central compartment (10), and it is even worse for deeper LNprRLN.

Intraoperative sentinel lymph node (SLN) biopsy for thyroid malignancy was first proposed in 1998 (11). Subsequently, there have been many attempts at SLN biopsy for thyroid cancer. Vital dye, lymphoscintigraphy, and a combined technique including vital dye and ${ }^{99 \mathrm{~m}} \mathrm{TC}$ nanocolloid particles have been described in several studies (12). However, the lymphatic drainage direction is not always predictable due to the complicated crosscommunication of lymphatic channels associated with the thyroid capsule. Up to now, the concept of SLN biopsy of thyroid carcinoma has not been widely accepted, but it does provide information for lymph node status, especially for patients with extensive lymph node metastasis who might benefit from the treatment with radioactive iodine (RAI). Therefore, our center has routinely carried out intraoperative lymph node biopsy during thyroid malignant neoplasm surgical procedures. Carbon nanoparticles $(\mathrm{CNs})$ have been applied as a novel lymphatic tracer in thyroid surgery in recent years, and there have been reports of SLN biopsy guided by CNs (13). The CNs can maintain SLN staining's durability compared to the methylene blue. It has increased the sensitivity and accuracy rates and reduced false-negative rate. There have been few reports on $\mathrm{CN}$-guided lymph node biopsy to evaluate LN-prRLN metastasis. Our present study aimed to provide a reference for evaluating the necessity of LN-prRLN dissection by combining patient clinicopathologic characteristics and intraoperative lymph node biopsy. We hypothesized that the intraoperative lymph nodes biopsy might predict $\mathrm{LN}$ prRLN metastasis in $\mathrm{cN} 0$ thyroid carcinoma patients and when an intraoperative biopsy indicates no lymph node metastasis, the LNs-prRLN are less likely to metastasize. Eventually, this would enable the avoidance of unnecessary LN-prRLN dissection during CLND. We present the following article in accordance with the STARD reporting checklist (available at http://dx.doi.org/10.21037/gs-20920).

\section{Methods}

\section{Patients}

We retrospectively analyzed consecutive patients diagnosed with PTC by US imaging and fine-needle aspiration (FNA), and the preoperative US showed no abnormal enlarged lymph nodes (cN0), who were admitted to the Center for Thyroid and Breast Surgery, Department of General Surgery, Xuanwu Hospital from January 2017 to November 2020. The patient's clinicopathologic features were comprehensively collected and analyzed. Participants were divided into metastatic LN-prRLN group (LN-prRLN positive group) and non-metastatic LN-prRLN group (LN-prRLN negative group) according to the LN-prRLN status at the postoperative pathologic examination. We 

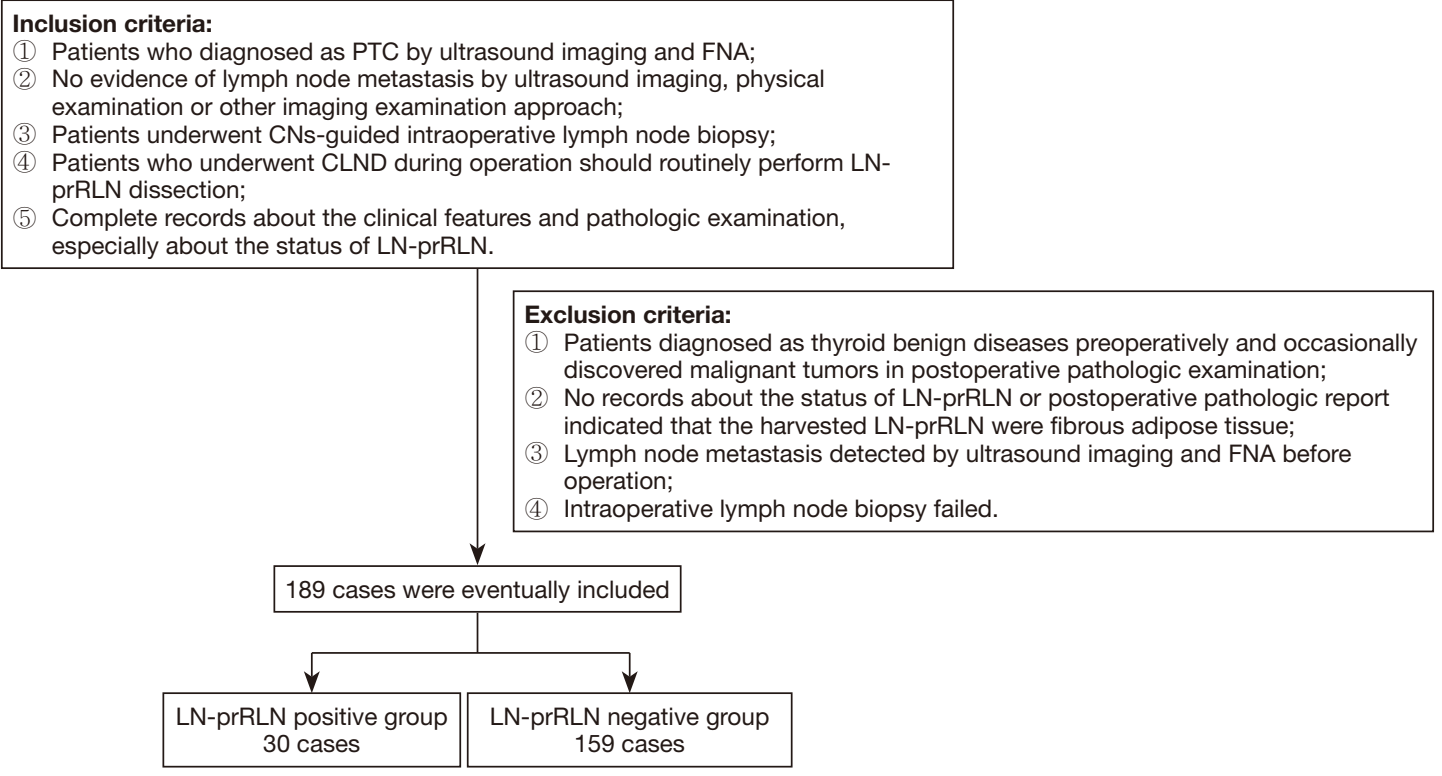

Figure 1 Flow chart for the study. PTC, papillary thyroid carcinoma; FNA, fine-needle aspiration; CNs, carbon nanoparticles; LN-prRLN, lymph nodes posterior to right recurrent laryngeal nerve; CLND, central lymph node dissection.

excluded patients with a preoperative clinical diagnosis of benign lesions. At the same time, postoperative pathological examination unexpectedly revealed thyroid carcinoma, those who underwent CLND but did not have a dedicated record of LN-prRLN, and those without a complete clinicopathologic case record were also excluded (Figure 1).

\section{Surgical procedure}

To evaluate the lymph node status intraoperatively, we routinely performed the intraoperative lymph node biopsy before the thyroid lobectomy. After the thyroid gland had been completely exposed, CNs were injected subcapsularly into the capsule of the isthmus and thyroid lobes ipsilateral to the tumors. The concentration of $\mathrm{CNs}$ suspension was $25 \mathrm{mg} / 0.5 \mathrm{~mL}$ (LUMMY, Chongqing, China), and $0.05-0.1 \mathrm{~mL}$ of $\mathrm{CNs}$ were injected at each location. The black stained lymphatic vessels and lymph nodes in the perithyroidal region were observed after about 5 minutes. The black-stained pre-laryngeal (Delphian) lymph nodes, pre-tracheal lymph nodes, and para-tracheal lymph nodes were then resected for intraoperative frozen pathological examination. Patients with unilateral lesions underwent thyroid lobectomy associated with ipsilateral CLND when the frozen pathological examination indicated no lymph node metastasis. Patients with bilateral lesions or a frozen pathological examination indicating lymph node metastasis underwent total thyroidectomy and bilateral CLND. When performing the right CLND, the right posterior laryngeal nerve lymph nodes were recorded and analyzed separately. All resected specimens were confirmed by pathological examination.

\section{Statistical analysis}

Data were analyzed with SPSS version 23.0 (IBM Corp., Armonk, NY, USA). The differences between the 2 groups of categorical variables were evaluated by Pearson's chi-square test, and the differences between the 2 groups of abnormally distributed continuous variables were calculated using the rank-sum test. Receiver operating characteristic (ROC) curves were used to find the cut-off values of LN-prRLN identified by intraoperative lymph node metastasis. Univariate and multivariate logistic regression was used to identify risk factors associated with LN-prRLN metastasis. Factors with statistical significance after screening by univariable regression analysis ( $\mathrm{P}$ value: entry 0.05 , removal 0.10 ) were put into the multivariable regression model. All data were analyzed anonymously and identified before analysis. A P value of $<0.05$ was considered statistically significant. 


\section{Ethics approval}

The study was approved by the Ethics Committee of Xuanwu Hospital, Capital Medical University based in Beijing, China \{20 April 2020, ID [2020]055\}. Participant data were kept confidential, and the details were presented such that the participants' identity cannot be ascertained. The requirement for informed consent was waived because of the retrospective nature of the study. All procedures performed in studies involving human participants were done so following the ethical standards of the institutional research committees and with the Declaration of Helsinki (as revised in 2013).

\section{Results}

\section{General characteristics}

A total of 236 consecutive patients diagnosed with thyroid papillary carcinoma with a comprehensive postoperative pathologic record of $L N$-prRLN were recruited for this study. Postoperative pathologic reports indicated that the harvested LN-prRLN were composed of fibrous adipose tissue in 23 cases. No intraoperative lymph node biopsy was performed in 24 cases due to central or lateral lymph node metastases discovered by the preoperative US and confirmed by FNA (cN1). Eventually, 189 participants (74 male and 115 female) were included in this study. The clinical and pathologic characteristics are summarized in Table 1. The overall incidence of LN-prRLN metastasis was $15.9 \%(30 / 189)$, and more younger patients and more metastatic lymph nodes after CLND were found among the LN-prRLN positive group $(\mathrm{P}=0.002)$.

\section{Intraoperative lymph node biopsy predicts the status of $L N$ - $\operatorname{prRLN}$}

The median and upper/lower quartile numbers of lymph nodes that were resected in $\mathrm{CN}$-guided intraoperative lymph node biopsy were $5[3,6]$, including $1[1,2]$ of Delphian lymph nodes, $1[1,2]$ of pre-tracheal lymph nodes, and 1 [1,2] of para-tracheal lymph nodes ipsilateral to the tumor. The univariate and multivariate regression analysis, including several potential risk factors previously reported, revealed that the number of metastatic lymph nodes during intraoperative lymph node biopsy was the potential predictor for LN-prRLN metastasis (Table 2).

To further study the predictive effect of intraoperative lymph node biopsy in LN-prRLN metastasis, the number of metastatic Delphian lymph nodes, pre-tracheal lymph nodes, and para-tracheal lymph nodes were calculated, respectively. More metastatic lymph nodes were discovered during intraoperative lymph node biopsy in participants with positive $\mathrm{LN}$-prRLN, including more metastatic Delphian lymph nodes, pre-tracheal lymph nodes, and paratracheal lymph nodes (Table 3).

Moreover, the ROC curve for predicting metastatic LNprRLN by the number of metastatic lymph nodes during intraoperative lymph nodes biopsy was generated. The Delphian lymph nodes, pre-tracheal lymph nodes, and paratracheal lymph nodes metastases could respectively predict the metastasis of LN-prRLN. However, it was only when these lymph node states were analyzed in combination that the area under the curve (AUC) could reach 0.7. The cut-off value was 0.5 , which illustrated that the number of metastatic lymph nodes $\geq 1$ might be the LN-prRLN metastasis threshold (Figure 2). The sensitivity was $80.0 \%$, and the specificity was $56.6 \%$. The positive predictive value was $25.8 \%$, while the negative predictive value was $93.8 \%$ (Table 4).

\section{Discussion}

In recent years, thyroid surgeons have given more attention to the LN-prRLN during the right CLND. As is known to all, PTC has a fairly strong tendency of regional lymph node metastasis, especially the lymph nodes located at the central compartment. A study published by Ito et al. revealed that $59 \%$ of the $4301 \mathrm{cN} 0$ PTC patients were finally diagnosed as $\mathrm{pN} 1 \mathrm{a}$ on postoperative pathological examination and that routine prophylactic CLND resulted in an excellent recurrence-free survival (14). Also, Kim et al. illustrated that CLND during the initial operation decreased the risk of local soft tissue and distant recurrences (3). Although there has not been a consensus for routine CLND, we have routinely performed CLND for PTC under the adequate protection of the RLNs and parathyroid glands to obtain a more accurate tumor pathological stage then guide follow-up treatment. However, traction on the RLN during the dissection of the LN-prRLN undoubtedly increases the risk of nerve injury and the operating time. Therefore, we need to determine whether patients staging in $\mathrm{cN} 0$ can benefit from LN-prRLN dissection at the risk of RLN injury during the routine prophylactic CLND.

According to published studies, the incidence of LNprRLN metastasis was $17.14-51.9 \%$, and several potential 
Table 1 Clinical characteristics in LN-prRLN positive and negative group [n(\%)]

\begin{tabular}{|c|c|c|c|c|}
\hline Variables & \multicolumn{2}{|c|}{ Status of LN-prRLN } & Statistic & $P$ value \\
\hline Age, years & $35.5(28.75,45.5)$ & $44(35,55)$ & -3.105 & 0.002 \\
\hline$<55$ & $28(93.3)$ & $118(74.2)$ & 5.249 & 0.022 \\
\hline$\geq 55$ & $2(6.7)$ & $41(25.8)$ & & \\
\hline Male & $16(53.3)$ & $58(36.5)$ & 3.010 & 0.083 \\
\hline Female & $14(46.7)$ & $101(63.5)$ & & \\
\hline \multicolumn{5}{|l|}{ Hashimoto's thyroiditis } \\
\hline Yes & $14(46.7)$ & $52(32.7)$ & 0.029 & 0.866 \\
\hline \multicolumn{5}{|l|}{ T stage } \\
\hline T1a & $16(53.3)$ & $102(64.2)$ & 1.259 & 0.262 \\
\hline $\mathrm{T} 1 \mathrm{~b}$ & $12(40.0)$ & 45 (28.3) & 1.640 & 0.200 \\
\hline T2 & $2(6.7)$ & $7(4.4)$ & 0.285 & 0.593 \\
\hline T3 & $0(0)$ & $5(3.1)$ & 0.969 & 0.325 \\
\hline Number of metastatic lymph nodes in total CLND & $5.5(3.0,9.0)$ & $1(0,4)$ & -5.039 & $<0.001$ \\
\hline \multicolumn{5}{|l|}{ RLN injury } \\
\hline Yes & $2(6.7)$ & $2(1.3)$ & 3.564 & 0.059 \\
\hline Right & $19(63.3)$ & $93(58.5)$ & 0.245 & 0.621 \\
\hline Left & $2(6.7)$ & $18(18)$ & 0.578 & 0.447 \\
\hline Bilateral & $9(30.0)$ & $48(30.2)$ & 0.000 & 0.984 \\
\hline
\end{tabular}

LN-prRLN, lymph nodes posterior to right recurrent laryngeal nerve; CLND, central lymph node dissection; RLN, recurrent laryngeal nerve.

predictors such as age, male gender, tumor diameter, capsular invasion, comorbid adenoma, Hashimoto's thyroiditis, and VIa compartment lymph nodes metastasis were considered to be significantly associated with $\mathrm{LN}$ prRLN metastasis (15-22). Our center's data revealed that the incidence of LN-prRLN metastasis was $15.9 \%$, but our multivariate analysis suggested that none of the indicators mentioned above were good predictors for the status of
LN-prRLN. We considered that the probable cause of this result is more participants with advanced stages such as lateral lymph node metastasis or T3 in previously published studies, so participants showed more progressive features. Also, in almost all of the published literature, the analyses were performed according to postoperative pathological or clinical data, and there was a lack of a preferred approach to guide surgical decision-making during the operation. 
Table 2 Univariate and Multivariate analysis of metastatic LN-prRLN with potential risk factors

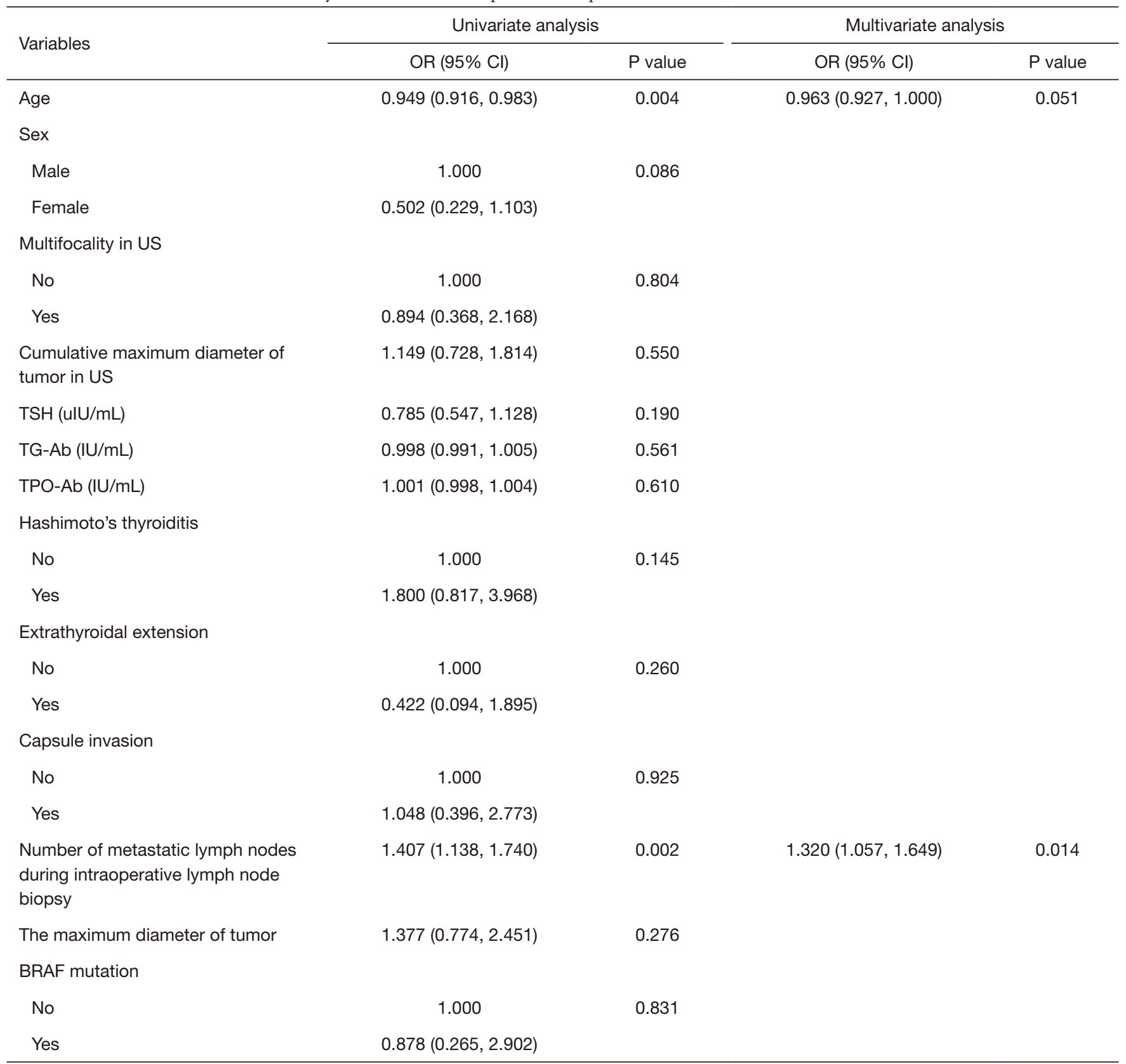

LN-prRLN, lymph nodes posterior to right recurrent laryngeal nerve; OR, odds ratio; Cl, confidence interval; US, ultrasound; TSH, thyroid stimulating hormone; TG-Ab, thyroglobulin antibody; TPO-Ab, thyroid peroxidase antibody; BRAF, B-type Raf kinase.

Hence, patients in the relatively early stages must find appropriate indicators to accurately evaluate the status of LN-prRLN pre- and intra-operatively to avoid nerve injury caused by unnecessary lymph node dissection.

Although SLN biopsy has not been widely accepted as a diagnostic method for lymph node metastasis of thyroid carcinoma like breast cancer, it provides information for lymph node status. The vital dye is one of the methods used to trace lymphatic drainage. The sensitivity was $57-100 \%$, specificity was $94-100 \%$, and the false-negative rate was 0-38\% (12). According to the published studies, the SLNs are mostly located at the central compartment $(23,24)$, and Delphian lymph nodes could even predict the lymph node metastasis alone (25). Accordingly, we selected Delphian, 
Table 3 Numbers of metastatic lymph nodes in intraoperative lymph nodes biopsy

\begin{tabular}{|c|c|c|c|c|}
\hline Variables & \multicolumn{2}{|c|}{ Status of LN-prRLN } & Statistic value & $P$ value \\
\hline $\begin{array}{l}\text { Total Number of metastatic lymph nodes in } \\
\text { sentinel lymph node biopsy }\end{array}$ & $0(0,2)$ & $2(1,4)$ & $-3.764^{a}$ & $<0.001$ \\
\hline Number of metastatic Delphian lymph nodes & $0(0,0)$ & $0(0,1)$ & $-2.885^{a}$ & 0.004 \\
\hline Number of metastatic pre-tracheal lymph nodes & $0(0,1)$ & $1(0,2)$ & $-3.634^{\mathrm{a}}$ & $<0.001$ \\
\hline
\end{tabular}

${ }^{\text {a }}$ Z value for Mann-Whitney $U$ test.

A

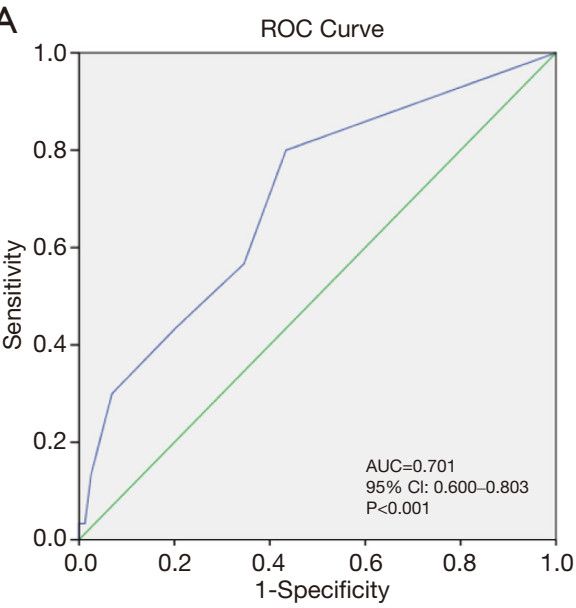

C

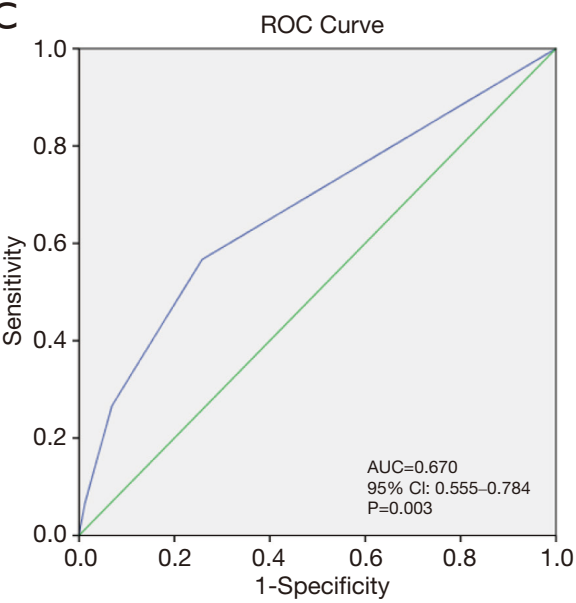

B

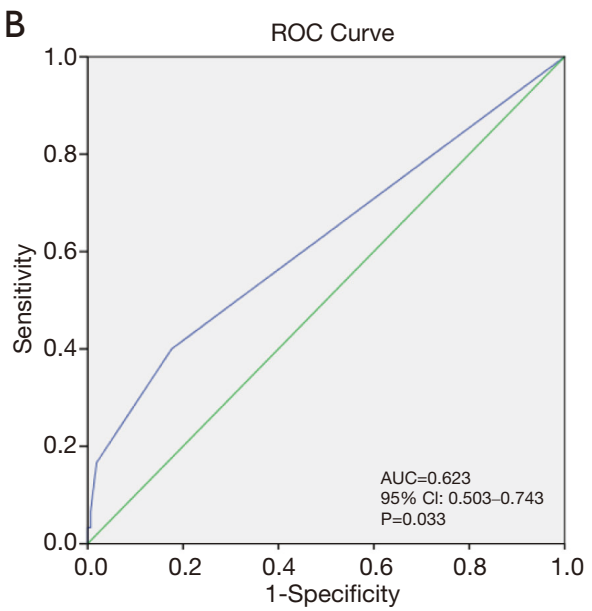

D

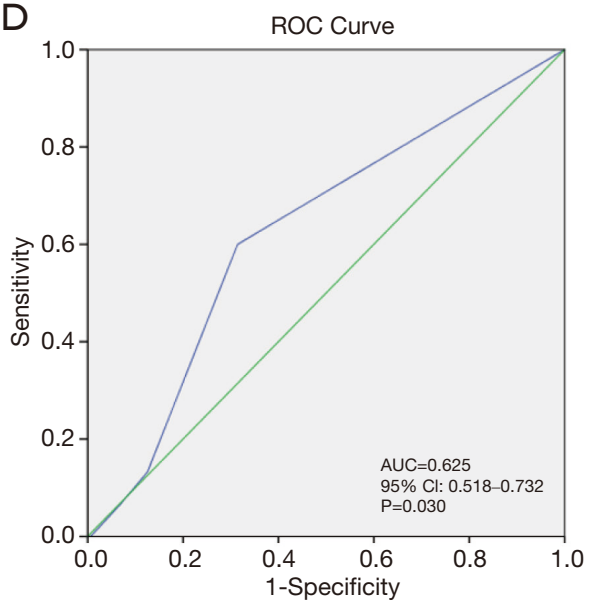

Figure 2 ROC for predicting metastatic LN-prRLN by number of metastatic intraoperative lymph nodes discovered intraoperatively. Our model performance and random guess performance are presented in the blue line and the green line, respectively. (A) Predicting metastatic LN-prRLN by the number of total metastatic intraoperative lymph nodes. (B) Predicting metastatic LN-prRLN by the number of metastatic Delphian lymph nodes. (C) Predicting metastatic LN-prRLN by the number of metastatic pre-tracheal lymph nodes. (D) Predicting metastatic LN-prRLN by the number of metastatic para-tracheal lymph nodes. ROC, receiver operating characteristic curve; LN-prRLN, lymph node metastases posterior to the right recurrent laryngeal nerve; AUC, area under the curve; CI, confidence interval. 
Table 4 The predictive value of intraoperative lymph nodes biopsy in LN-prRLN metastasis [n(\%)]

\begin{tabular}{lcc}
\hline \multirow{2}{*}{ Status of intraoperative lymph nodes biopsy } & Status of LN-prRLN & Negative $(\mathrm{n}=159)$ \\
\cline { 2 - 4 } & Positive $(\mathrm{n}=30)$ & $69(74.2)$ \\
Positive $(\mathrm{n}=93)$ & $24(25.8)$ & $90(93.8)$ \\
\hline Negative $(n=96)$ & $6(6.2)$ & 90 \\
\hline
\end{tabular}

pre-tracheal, and para-tracheal lymph nodes as target lymph nodes to cover as much of the lymphatic drainage as possible and ensure the simplicity of lymph node biopsy. Surprisingly, our results demonstrated that the number of metastatic lymph nodes in intraoperative frozen section examination was a potential predictor of LN-prRLN metastasis. Notably, the false-negative rate was fairly low (6.2\%), which suggested that LN-prRLN dissection might not be necessary for $\mathrm{cN} 0$ patients when the lymph nodes biopsy did not indicate metastasis. Zhu et al. drew a similar conclusion in a retrospective study including 592 patients. Their results considered that the Delphian lymph node metastasis and number of metastatic pre-tracheal and paratracheal lymph nodes were independent predictors of $\mathrm{LN}$ prRLN metastasis. However, the difference was that the intraoperative frozen section examination of the lymph nodes was performed after the entire CLND in their study (17), which probably means that they had to wait for the pathologic report after the thyroid lobectomy and CLND. In comparison, the SLN procedure can be carried out before the thyroid lobectomy and the entire CLND. After the above procedure, surgeons can immediately decide whether to perform LN-prRLN dissection or even thyroidectomy with bilateral central lymph node dissection according to the intraoperative frozen section examination.

With the development of personalized diagnosis and treatment concepts, personalized surgical volume and surgical approach are important in increasingly patientcentered decision-making. The ATA recommendation is to abandon prophylactic CLND in T1 or T2, noninvasive, and cN0 PTC patients. Patients with clinical N0 or $\leq 5$ pathologic $\mathrm{N} 1$ micro-metastases $(<0.2 \mathrm{~cm}$ in largest dimension) were classified as ATA low-risk group, which predicted a recurrence rate below 5\% (26). However, according to the published literature, the incidence of lymph node metastasis in $\mathrm{cN} 0$ patients ranged from $25-82.3 \%$ (27), similar to the incidence $(64.02 \%)$ in our series. Moreover, our postoperative pathologic examination results indicated that micro-metastases but no macro-metastases in lymph nodes were found in 5 cases, and micro-metastases in $\mathrm{LN}$ -
prRLN were found in only 1 case. Therefore, in our clinical practice, prophylactic CLND is still routinely carried out due to the inconsistency between the clinical nodal staging and pathological staging. Because of the small number of cases, it is probably difficult to explain micro-metastasis clinical significance in our present study. However, for all of the 5 cases with micro-metastasis, there were no positive lymph nodes detected during intraoperative lymph node biopsy. Patients with no metastatic lymph nodes during intraoperative biopsy might still represent a low risk of LNprRLN metastasis. In conclusion, the intraoperative lymph node biopsy method could provide reference for the risk of LN-prRLN metastasis to develop a personalized surgical volume.

Our present research was limited in that it was a singlecenter retrospective study, and the sample size was relatively small. Therefore, a multi-center study with a larger number of cases is necessary to confirm further the accuracy and feasibility of $\mathrm{CN}$-guided intraoperative lymph node biopsy in predicting the status of LN-prRLN. Moreover, the follow-up indicators such as postoperative thyroglobulin levels, thyroid function, nodal recurrence rate, and diseasefree survival were not evaluated in our present study. To clarify whether LN-prRLN dissection can be avoided in patients with no metastasis in intraoperative lymph node biopsy, future randomized controlled trials to evaluate the oncological outcomes are necessary to validate our conclusions.

\section{Conclusions}

The number of metastatic lymph nodes in $\mathrm{CN}$-guided intraoperative lymph node biopsy, including pre-laryngeal (Delphian) lymph nodes, pre-tracheal lymph nodes, and para-tracheal lymph nodes ipsilateral to the tumor, were potential predictors of LN-prRLN metastasis in $\mathrm{cN} 0$ thyroid carcinoma patients. Patients with no metastatic lymph nodes during intraoperative biopsy might represent a low risk of LN-prRLN metastasis. However, for patients with $\mathrm{cN} 0$ and negative lymph nodes in an intraoperative 
biopsy, further randomized controlled trials are necessary to assess the prognostic outcomes and validate whether the dissection of LN-prRLN could be avoided during CLND.

\section{Acknowledgments}

We thank Dr. Yutian Yin (Department of Radiation Oncology, Xijing Hospital, Xi'an, China) for the professional guidance for statistics and we thank the English language editors: J. Jones and J. Chapnick from AME Editing Service for revising the language of this article.

Funding: This work was supported by the Beijing Municipal Health System Academic Leaders of High-level Health Personnel Program, China (2011-2-28).

\section{Footnote}

Reporting Checklist: The authors have completed the STARD reporting checklist. Available at http://dx.doi.org/10.21037/ gs-20-920

Data Sharing Statement: Available at http://dx.doi. org/10.21037/gs-20-920

Peer Review File: Available at http://dx.doi.org/10.21037/gs20-920

Conflicts of Interest: All authors have completed the ICMJE uniform disclosure form (available at http://dx.doi. org/10.21037/gs-20-920). The authors have no conflicts of interest to declare.

Ethical Statement: The authors are accountable for all aspects of the work in ensuring that questions related to the accuracy or integrity of any part of the work are appropriately investigated and resolved. The study was approved by the Ethics Committee of Xuanwu Hospital, Capital Medical University, based in Beijing, China $\{20$ April 2020, ID [2020]055\}. Participant data were kept confidential, and the details were presented such that the participants' identity cannot be ascertained. The requirement for informed consent was waived because of the retrospective nature of the study. All procedures performed in studies involving human participants were done so following the ethical standards of the institutional research committee and with the Declaration of Helsinki (as revised in 2013).
Open Access Statement: This is an Open Access article distributed in accordance with the Creative Commons Attribution-NonCommercial-NoDerivs 4.0 International License (CC BY-NC-ND 4.0), which permits the noncommercial replication and distribution of the article with the strict proviso that no changes or edits are made and the original work is properly cited (including links to both the formal publication through the relevant DOI and the license). See: https://creativecommons.org/licenses/by-nc-nd/4.0/.

\section{References}

1. $\mathrm{Li} \mathrm{F}, \mathrm{Wu} \mathrm{Y}$, Chen $\mathrm{L}$, et al. Evaluation of clinical risk factors for predicting insidious right central and posterior right recurrent laryngeal nerve lymph node metastasis in papillary thyroid microcarcinoma patients (cN0): experience of a single center. Ann Transl Med 2019;7:8.

2. Luo X, Wang J, Xu M, et al. Risk model and risk stratification to preoperatively predict central lymph node metastasis in papillary thyroid carcinoma. Gland Surg 2020;9:300-10.

3. Kim H, Kim TH, Choe JH, et al. Patterns of Initial Recurrence in Completely Resected Papillary Thyroid Carcinoma. Thyroid 2017;27:908-14.

4. Salem FA, Bergenfelz A, Nordenstrom E, et al. Central lymph node dissection and permanent hypoparathyroidism after total thyroidectomy for papillary thyroid cancer: population-based study. Br J Surg 2020.

5. Ling Y, Zhao J, Zhao Y, et al. Role of intraoperative neuromonitoring of recurrent laryngeal nerve in thyroid and parathyroid surgery. J Int Med Res 2020;48:300060520952646.

6. Shen WT, Ogawa L, Ruan D, et al. Central neck lymph node dissection for papillary thyroid cancer: comparison of complication and recurrence rates in 295 initial dissections and reoperations. Arch Surg 2010;145:272-5.

7. Haugen BR, Alexander EK, Bible KC, et al. 2015 American Thyroid Association Management Guidelines for Adult Patients with Thyroid Nodules and Differentiated Thyroid Cancer: The American Thyroid Association Guidelines Task Force on Thyroid Nodules and Differentiated Thyroid Cancer. Thyroid 2016;26:1-133.

8. Takami H, Ito Y, Okamoto T, et al. Therapeutic strategy for differentiated thyroid carcinoma in Japan based on a newly established guideline managed by Japanese Society of Thyroid Surgeons and Japanese Association of Endocrine Surgeons. World J Surg 2011;35:111-21. 
9. Agrawal N, Evasovich MR, Kandil E, et al. Indications and extent of central neck dissection for papillary thyroid cancer: An American Head and Neck Society Consensus Statement. Head Neck 2017;39:1269-79.

10. Khokhar MT, Day KM, Sangal RB, et al. Preoperative High-Resolution Ultrasound for the Assessment of Malignant Central Compartment Lymph Nodes in Papillary Thyroid Cancer. Thyroid 2015;25:1351-4.

11. Kelemen PR, Van Herle AJ, Giuliano AE. Sentinel lymphadenectomy in thyroid malignant neoplasms. Arch Surg 1998;133:288-92.

12. Garau LM, Rubello D, Ferretti A, et al. Sentinel lymph node biopsy in small papillary thyroid cancer. A review on novel surgical techniques. Endocrine 2018;62:340-50.

13. Hao RT, Chen J, Zhao LH, et al. Sentinel lymph node biopsy using carbon nanoparticles for Chinese patients with papillary thyroid microcarcinoma. Eur J Surg Oncol 2012;38:718-24.

14. Ito Y, Miyauchi A, Masuoka H, et al. Excellent Prognosis of Central Lymph Node Recurrence-Free Survival for cNOM0 Papillary Thyroid Carcinoma Patients Who Underwent Routine Prophylactic Central Node Dissection. World J Surg 2018;42:2462-8.

15. Wang Y, Wang D, Chen L, et al. A Comparison of Two Operation Methods Revealed the Risk Factors and the Necessity of LN-prRLN Dissection in Papillary Thyroid Carcinoma: A Retrospective Cohort Study in FUSCC. Int J Endocrinol 2020;2020:7162793.

16. Zou M, Wang YH, Dong YF, et al. Clinical and sonographic features for the preoperative prediction of lymph nodes posterior to the right recurrent laryngeal nerve metastasis in patients with papillary thyroid carcinoma. J Endocrinol Invest 2020;43:1511-7.

17. Zhu J, Huang R, Hu D, et al. Individualized Prediction Of Metastatic Involvement Of Lymph Nodes Posterior To The Right Recurrent Laryngeal Nerve In Papillary Thyroid Carcinoma. Onco Targets Ther 2019;12:9077-84.

18. Hou J, Shan H, Zhang Y, et al. Risk factors of metastasis to the lymph nodes posterior to the right recurrent laryngeal nerve in papillary thyroid carcinoma. Eur Arch

Cite this article as: Ling Y, Zhang L, Li K, Zhao Y, Zhao J, Jia L, Wang Y, Kang H. Carbon nanoparticle-guided intraoperative lymph node biopsy predicts the status of lymph nodes posterior to right recurrent laryngeal nerve in $\mathrm{cN} 0$ papillary thyroid carcinoma. Gland Surg 2021;10(5):1554-1563. doi: 10.21037/gs-20-920
Otorhinolaryngol 2020;277:881-6.

19. Luo Y, Xu XC, Shen J, et al. Model of lymph node metastasis posterior to the right recurrent laryngeal nerve in papillary thyroid carcinoma. Cancer Manag Res 2018;10:2449-55.

20. Yuan J, Li J, Chen X, et al. Predictors of lymph nodes posterior to the right recurrent laryngeal nerve metastasis in patients with papillary thyroid carcinoma: A retrospective study. Medicine (Baltimore) 2017;96:e7908.

21. Liu Z, Sun M, Xiao Y, et al. Predictors of metastasis to lymph nodes posterior to the right recurrent laryngeal nerve in differentiated thyroid carcinoma: A prospective study. Asian J Surg 2017;40:270-7.

22. Pinyi Z, Bin Z, Jianlong B, et al. Risk factors and clinical indication of metastasis to lymph nodes posterior to right recurrent laryngeal nerve in papillary thyroid carcinoma: a single-center study in China. Head Neck 2014;36:1335-42.

23. Cabrera RN, Chone CT, Zantut-Wittmann D, et al. Value of sentinel lymph node biopsy in papillary thyroid cancer: initial results of a prospective trial. Eur Arch Otorhinolaryngol 2015;272:971-9.

24. Kaczka K, Luks B, Jasion J, et al. Sentinel lymph node in thyroid tumors - own experience. Contemp Oncol (Pozn) 2013;17:184-9.

25. Huang J, Sun W, Zhang H, et al. Use of Delphian lymph node metastasis to predict central and lateral involvement in papillary thyroid carcinoma: A systematic review and meta-analysis. Clin Endocrinol (Oxf) 2019;91:170-8.

26. Hong YR, Lee SH, Lim DJ, et al. The stratification of patient risk depending on the size and ratio of metastatic lymph nodes in papillary thyroid carcinoma. World J Surg Oncol 2017;15:74.

27. Sun W, Lan X, Zhang H, et al. Risk Factors for Central Lymph Node Metastasis in CN0 Papillary Thyroid Carcinoma: A Systematic Review and Meta-Analysis. PLoS One 2015;10:e139021.

(English Language Editors: J. Jones and J. Chapnick) 